\title{
The undefined anatomical variations of the deltoid ligament bundles: a cadaveric study
}

\author{
Marco Zamperetti ${ }^{1}$ \\ Matteo Guelfi ${ }^{2}$ \\ Carlo Biz ${ }^{1}$ \\ Andrea Pantalone ${ }^{2}$ \\ Vincenzo Salini ${ }^{2}$ \\ Xavier Martin Oliva ${ }^{3}$ \\ Pietro Ruggieri' \\ Rosa Maria Mirapeix ${ }^{4}$
}

1 Orthopaedic Clinic, Department of Surgery, Oncology and Gastroenterology DiSCOG, University of Padua, Padua, Italy

2 Orthopaedic and Traumatology Unit, Department of Medicine and Science of Aging, University "G. d'Annunzio", Chieti-Pescara, Chieti, Italy

3 Department of Anatomy and Human Embryology, Faculty of Medicine, University of Barcelona, Barcelona, Spain

4 Unit of Anatomy and Human Embriology, Universitat Autónoma de Barcelona, Barcelona, Spain

Corresponding author:

Carlo Biz

Orthopaedic Clinic

Department of Surgery, Oncology and

Gastroenterology DiSCOG

University of Padua, Padua, Italy

E-mail: carlo.biz@unipd.it

\section{Summary}

Introduction: Recently, Cromeens proposed a new interpretation of the deltoid ligament (DL) using its attachment sites to define individual components. The aim of this cadaveric study was to evaluate the reproducibility of this new classification and investigate anatomical variations of the ligament to contribute to reaching an evidencebased consensus regarding its ligamentous constituent bands, their origins and insertions.

Methods: The classification was applied to study only four components of the DL: the tibiocalcaneonavicular (TCN), the superficial posterior tibiotalar (SPTT), the deep anterior tibiotalar (dATT) and the deep posterior tibiotalar (dPTT) ligaments. Fifteen fresh frozen ankles were dissected and the deltoid ligament components de- fined by their attachment sites and measured on the three axes.

Results: The TCN, sPTT and dPTT ligaments were identified in all of the specimens. The TCN was the widest and longest ligament with variable thickness. The SPTT ligament was the second thicker and longer. The dATT ligament, the smaller and thinner, was not identified in 3 specimens. The dPTT ligament was the second wider, which in 3 cadavers presented an accessory bundle, the deep intermedial tibiotalar ligament (dITT ligament).

Conclusions: Cromeens's attachment-based classification was proved to be simple, rational and reproducible in allowing the methodological description of the different bands of the DL. Our findings showed that the dITT ligament must be considered a secondary bundle of the dPTT ligament, which was not always identified in all specimens.

Level of evidence: IV.

\section{Clinical relevance}

The anatomical data regarding the deltoid ligament bands and their variations described in this study should promote correct management of ankle sprains in cases of deltoid ligament injury or instability, often trivialized, addressing the proper treatment: conservative or operative.

KEY WORDS: anatomy, ankle injuries, ankle joint, foot, joint instability.

\section{Introduction}

The deltoid ligament (DL) is a strong, broad triangular ligament composed of different fascicles of variable presence and contribution of its superficial and deep layers, separated by a fat pad ${ }^{1-4}$. Previous qualitative and quantitative observations have shown how this ligament, by its origin from the medial malleolus spanning towards insertion in the navicular, talus and calcaneus, is an important stabilizer not only of the medial tibiotalar joint, but also the entire tibiotalocalcaneal joint complex ${ }^{1,5,6}$. Functionally, the superficial layer restricts the tendency of the talus to shift into a valgus ankle position and translate anterolaterally, resisting eversion of the hindfoot, while the deep layer is the primary restraint to external rotation of the 
talus $^{7}$. Further, according to Ziai et al., it also has a lateral stabilizing role during the movement of supination-inversion in cases of injuries of the anterior talofibular ligament and the calcaneofibular ligament ${ }^{8}$. Isolated DL lesions, accounting for nearly 5 to $15 \%$ of ankle sprains in the active population ${ }^{9-15}$, are not as common as lateral ankle lesions. However, if not adequately diagnosed and treated, they can lead to chronic medial or multidirectional ankle instability. Often, they occur with concomitant lateral ankle sprains during fractures of the lateral malleolus ${ }^{14}$ and in association with syndesmosis lesions, talar chondropathy and posterior tibial tendon pathology ${ }^{14-20}$. Although the majority of these sprains, being mild to moderate, can be treated non-operatively ${ }^{4}$, the acute complete lesions may result in significant time loss and disability in the general and athletic population because of consequent symptomatic medial prolonged instability or pronation deformity, which often require surgical repair or reconstruction ${ }^{14}$.

Obviously, for the success of all operative techniques, both open or arthroscopic, scientific basis and adequate knowledge of the normal morphology of the DL are mandatory $20-22$. However, due to the difficult isolation of the bundles, the interpretations of the anatomy of this ligament are so numerous and controversial that the evolution of the deltoid injury pattern lacks continuity in the literature, and the heterogeneous nomenclature given to the different bundles has not yet been discussed and accepted by the Federative Committee of Anatomical Terminology 5,23 .

Recently, Cromeens et al. ${ }^{24}$ proposed a new interpretation of the medial collateral ligament complex using their attachment sites as a guide to identify and describe five individual components: the tibiocalcaneonavicular (TCN), a union of the traditional tibionavicular, tibiospring, tibiocalcaneal, superomedial calcaneonavicular and medioplantar oblique ligaments; the superficial posterior tibiotalar (sPTT), the deep posterior tibiotalar (dPTT), the deep anterior tibiotalar ligament (dATT) and the inferoplantar longitudinal ligaments (iPL). This classification was considered by the Authors more functional and reproducible with respect to the traditional classification, but overly simplified in joining together in a single ligament the TCN, the traditional components of the spring ligament (superomedial and medioplantar ligament) and DL (tibionavicular, tibiospring and tibiocalcaneal ligament). However, in accordance with several Authors ${ }^{21,25,26}$, we believe that, although the spring ligament (SL) is commonly described to be connected with the DL, it should be considered independent anatomically and regarded as part of the spring ligament complex.

Hence, the purpose of this cadaveric study was to evaluate the reproducibility of this new classification excluding the SL (which includes the superomedial, the medioplantar and the iPL ligaments) and investigate anatomical variations by an in-depth description of the DL. The goal is to contribute to reaching an evidence-based consensus regarding the DL's ligamentous constituent bands, their origins and insertions.

\section{Material and methods}

Fifteen non-paired, fresh-frozen cadaveric adult ankle specimens, amputated at the level of the distal tibia $4 \mathrm{~cm}$ from the ankle joint line, were dissected. They were 8 right ankles and 7 left ones, and based on necropsy reports, acquired from 9 females and 6 males, all Caucasian, with a mean age of 75.87 years (range 65-92 years), a mean foot length of 23 $\pm 1.7 \mathrm{~cm}$ (the average adult foot length is $24.7 \pm 1.1$ $\mathrm{cm})^{21}$. No history of injury was reported and any deformities or signs of possible previous ankle surgery or trauma were present in the specimens selected for the study. All specimens were stored at $-10^{\circ} \mathrm{C}$, individually wrapped in cotton shrouds and placed in sealable plastic bags until dissection took place. They were obtained through the body donation programme of the universities of two of the Authors (R.M.M contributed with 12 specimens and X.M.O. with 3). Before death, all of the donors had expressed their consent of donating their bodies for anatomical education and study according to domestic law. The present study was conducted by all Authors ethically according to international standards as required by the Journal ${ }^{27}$.

After thawing, the specimens were placed on the dissection table with the foot plant resting on the table, the ankle in neutral position and the tibia and fibula secured to a stabilizing tower with rigid screws. The ankle specimens were dissected under magnification first removing the skin and crural fascia; then, by blunt dissection, the tibialis posterior and the flexor digitorum longus tendon, the neurovascular bundle and the flexor hallucis longus tendon were removed. Great attention was paid to remove the fibrous tendon sheath of the posterior tibialis tendon and flexor digitorum tendon strongly attached to the ligament below. Subsequently, the DL of each specimen was isolated removing the capsule anteriorly and posteriorly.

The deltoid bundles were defined by their continuous areas of attachment and measured on the three axes, length, thickness and width, using an electronic digital calliper ( $0.01 \mathrm{~mm}$ resolution, $0.02 \mathrm{~mm}$ accuracy). The location of the insertion points and the course of each ligament were marked with pins. The length was measured from pin to pin, with the ankle in neutral position; the thickness was measured at the mean point of the bundle; the width was measured at the proximal $(\mathrm{Pi})$ and distal $(\mathrm{Di})$ insertion. All measurements were taken three times each by two of the Authors (M.Z. and M.G.) and their mean values were calculated.

\section{Statistical analysis}

Statistical analyses were performed by an independent statistician (M.B.) from the Department of Statistics of our university using SAS 9.2 (SAS Institute Inc., Cary, NC, USA) for Windows. The comparison of patients' genders was performed with the unpaired ttest for the quantitative variables analysed (length, 
thickness and width) of each ligamentous component (TCN, sPTT, dPTT, dATT and dITT; their proximal and distal insertions). Statistical significance was set at $p<0.05$.

\section{Results}

The four main bundles that contribute to the DL complex as described by the Cromeens classification were identified and isolated in all fifteen specimens dissected except for the dATT ligament, which was not observed in three specimens. We referred to them as the tibiocalcaneonavicular (TCN), the superficial posterior tibiotalar (sPTT), the deep posterior tibiotalar (dPTT), the deep anterior tibiotalar ligament (dATT) "and the inferoplantar longitudinal ligaments (iPL)". The results of linear measurements for each component's perimeter and thickness are shown in Table I. Following the morphological description of the individual components, we identified a superficial (Fig. 1a) and deep layer (Fig. 2a). The superficial layer includes the TCN and SPTT ligament, while the deep layer includes the dPTT and the dATT ligament. A secondary bundle that we called the deep intermediate tibiotalar ligament (dITT) was identified in the deep layer. In almost all of our specimens there was no statistically significant difference between males and females with respect to the difference variables (length, width and thickness) measured for each bundle analysed except for the length of the SPTT ligament, which presented a low statistical significance $(p=0.0409)$.

\section{Superficial layers of the deltoid ligament}

Tibiocalcaneonavicular ligament (TCN)

The tibiocalcaneonavicular ligament is the largest and most complicated component of the DL and includes the tibiocalcaneal, tibionavicular and tibiospring ligaments. It was the widest ligament (mean Pi $17.98 \pm$
$2.11 \mathrm{~mm}$, mean Di $40.88 \pm 5.29 \mathrm{~mm})$, the longest (mean $33.51 \pm 2.52 \mathrm{~mm}$ ) and most complex to analyse (Fig. 1b). It had a variable thickness: the tibionavicular part was the thinnest (mean $0.87 \pm 0.06$ $\mathrm{mm}$ ), the spring-calcaneal part the thickest (mean 1.5 $\pm 0.41 \mathrm{~mm}$ ). It had a delta shaped fascicle with the proximal insertion attached to the antero-medial surface of the anterior colliculus of the tibial malleolus, the distal insertion attached to the medial third of the dorsal surface and posteriorly to the tuberosity of the navicular bone, and subsequently attached to the superior surface of the SL and to the superomedial surface of the sustentaculum tali. Its isolation was particularly difficult because it was hard to distinguish from the anterior joint capsule and because the tendon sheets of the posterior tibial and flexor digitorum longus muscles were strongly attached to the ligament. These three large attachment sites created a continuous band of tissue resembling a sling surrounding the head of the talus and will henceforth be referred to as the tibiocalcaneonavicular ligament. This ligament was present in all specimens.

\section{Superficial posterior tibiotalar ligament (sPTT)}

The superficial posterior tibiotalar ligament was easy to differentiate from the TCN. It was the second longest (mean $19.72 \pm 4.08 \mathrm{~mm}$ ) and thicker (mean $1.27 \mathrm{~mm} \pm 0.23 \mathrm{~mm}$ ) and the third widest (mean Pi $10.29 \pm 1.38 \mathrm{~mm}$, mean Di $12.38 \pm 1.56$ ). The tibial insertion attached from the intercollicular groove to the anteromedial surface of the posterior colliculus surface; the distal insertion attached from the medial surface of the posteromedial talar tuberculus to the supero-posterior surface of the substentaculum tali (Fig. 1c). The distal insertion to the talus was always present, while 8 cases presented a calcaneal insertion to the sustentaculum tali. A thin fat pad between the superficial and deep layer was present. The tibial attachment was the largest site followed by the talar attachment and the calcaneal attachment when present.

Table I. Linear measurements for each component studied.

\begin{tabular}{lllll}
\hline Bundles & $\begin{array}{l}\text { Proximal Insertion } \\
\text { mm; SD (range) }\end{array}$ & $\begin{array}{l}\text { Distal Insertion } \\
\text { mm; SD (range) }\end{array}$ & $\begin{array}{l}\text { Lenght } \\
\text { mm; SD (range) }\end{array}$ & $\begin{array}{l}\text { Thickness } \\
\text { mm; SD (range) }\end{array}$ \\
\hline TCN & $17.98 \pm 2.11(14.50-23.02)$ & $40.88 \pm 5.29(32.99-45.34)$ & $33.51 \pm 2.52(29.06-36.43)$ & $\begin{array}{l}0.87 \pm 0.06(0.81-1.00)^{*} \\
1.50 \pm 0.41(1.13-2.2)^{\circ}\end{array}$ \\
sPTT & $10.29 \pm 1.38(8.33-13.34)$ & $12.38 \pm 1.56(10.04-14.79)$ & $15.72 \pm 4.08(15.12-25.04)$ & $1.27 \pm 0.23(1.00-1.8)$ \\
dATT & $7.50 \pm 1.85(4.83-11.56)$ & $7.70 \pm 1.21(6.11-8.88)$ & $12.02 \pm 1.61(8.66-14.34)$ & $0.96 \pm 0.13(0.78-1.27)$ \\
dPTT & $12.49 \pm 1.67(9.80-15.34)$ & $15.54 \pm 2.17(13.33-20.34)$ & $12.63 \pm 2.28(8.4-15.12)$ & $1.14 \pm 0.23(0.84-1.54)$ \\
dITT & $7.90 \pm 0.46(7.40-8.30)$ & $8.39 \pm 0.58(8.05-9.06)$ & $12.67 \pm 2.08(11.00-15.00)$ & $1.22 \pm 0.36(0.80-1.44)$ \\
\hline
\end{tabular}

$\mathbf{T C N}=$ Tibiocalcaneonavicular ligament; $\mathbf{s P T T}=$ Superficial posterior tibio talar ligament; $\mathbf{d A T T}=$ Deep anterior tibio talar ligament; $\mathbf{d P T T}=$ Deep posterior tibio talar ligament; dITT= Deep intermedial tibio talar ligament; ${ }^{*}=$ Tibio-navicolar part of the TCN; ${ }^{\circ}=$ Spring-calcaneal part of the TCN. 


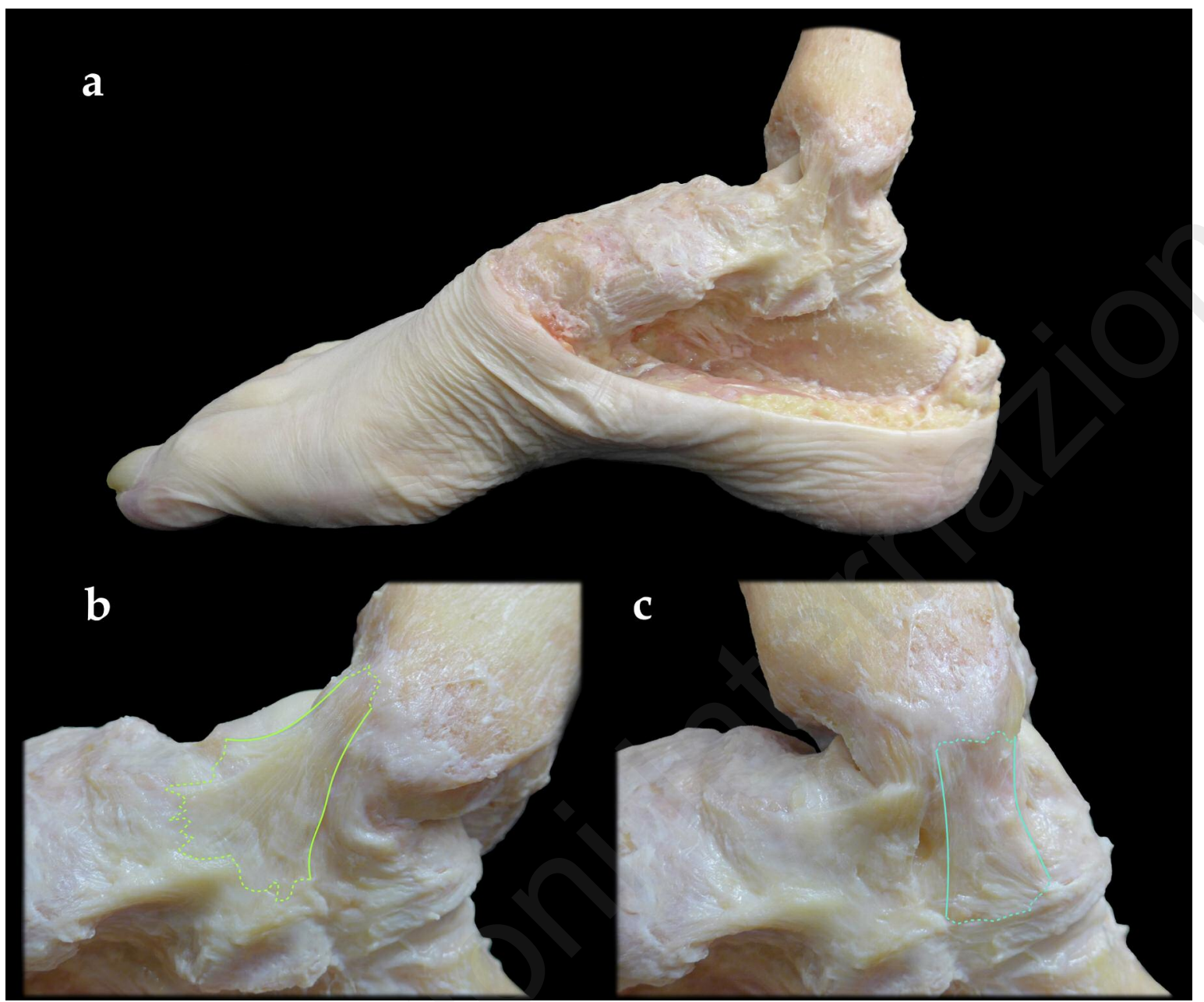

Figure 1. a) Medial ankle anatomic dissection (osteoarticular layer) showing the Superficial layer; b) TCN ligament, spreading from the anterior colliculus of the tibial malleolus to the navicular bone, the spring ligament and the substentaculm tali; c) SPTT ligament, inserted proximally between the intercollicular groove and the posterior colliculus and distally to the medial surface of the talus, occasionally crossing over the subtalar joint.

\section{Deep layer of the deltoid ligament}

Deep anterior tibiotalar ligament (dATT)

The deep anterior tibiotalar ligament was the smallest of the four principal bundles (Fig. 2b). It had a rectangular shaped fascicle with a mean width $\mathrm{Pi} 7.5 \pm 1.85$ $\mathrm{mm}$, Di $7.7 \pm 1.21 \mathrm{~mm}$, a mean thickness of $0.96 \pm$ $0.13 \mathrm{~mm}$ and a mean length of $12.02 \pm 1.61 \mathrm{~mm}$. The tibial insertion included the apex and the posterior part of the anterior colliculus; the distal insertion attached on the medial surface of the talus anterior to the dPTT insertion. In three cases it was not possible to identify.

Deep posterior tibiotalar ligament (dPTT)

The deep posterior tibiotalar ligament has a rectangular shaped fascicle and was present in all specimens. It was the second widest ligament (Pi $12.49 \mathrm{~mm} \pm$ 1.67 , Di $15.54 \mathrm{~mm} \pm 2.17$ ). It had a mean thickness of $1.14 \mathrm{~mm} \pm 0.23$ and a mean length of $12.63 \mathrm{~mm} \pm$
2.28 (Fig. 2b). The proximal tibial insertion attached from the intercollicular groove to the anterior part and apex of the posterior colliculus, descended posteriorly obliquely and attached under the articular facet of the medial malleolus. The talar attachment occurred just below the facies malleolaris medialis.

\section{Deep intermedial tibiotalar ligament (dITT)}

The deep intermedial tibiotalar ligament was present in only 3 cases. It had a mean thickness of $1.22 \mathrm{~mm}$ \pm 0.36 , a mean length of $12.67 \mathrm{~mm} \pm 2.08$ and a mean width of Pi $7.9 \mathrm{~mm} \pm 0.46$, Di $8.39 \mathrm{~mm} \pm 0.58$ (Fig. 3). When the dITT was present, the proximal insertions of dPTT were more posterior and limited. The tibial insertion attached from the posterior part of the intercollicular groove to the anterior part of the posterior colliculus, descended vertically and attached to the talus under the anterior part of the facies malleolaris medialis, just anterior to the talar attachment of the dPTT. 


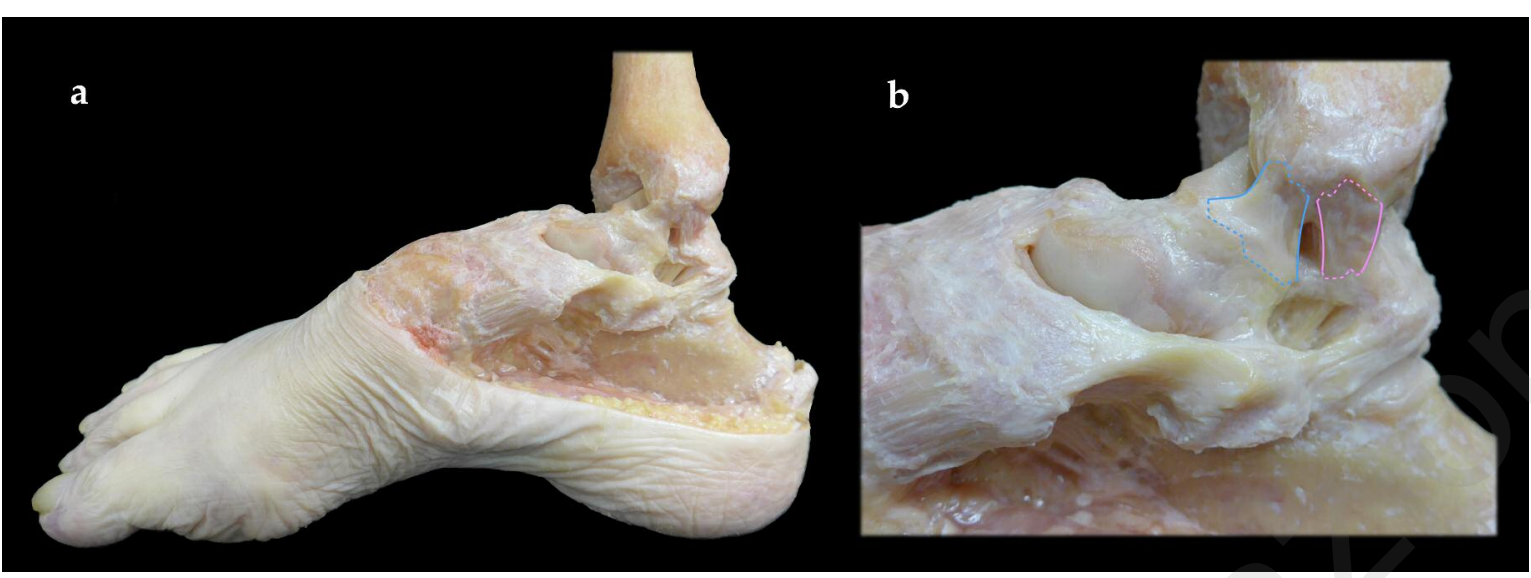

Figure 2. a) Anatomic view of the Deep layer; b) the dATT ligament (blue margin) inserts from the anterior colliculus of the tibia to the medial surface of the talus; the dPTT ligament (pink margin) runs from the anterior colliculus to the medial surface of the talus.

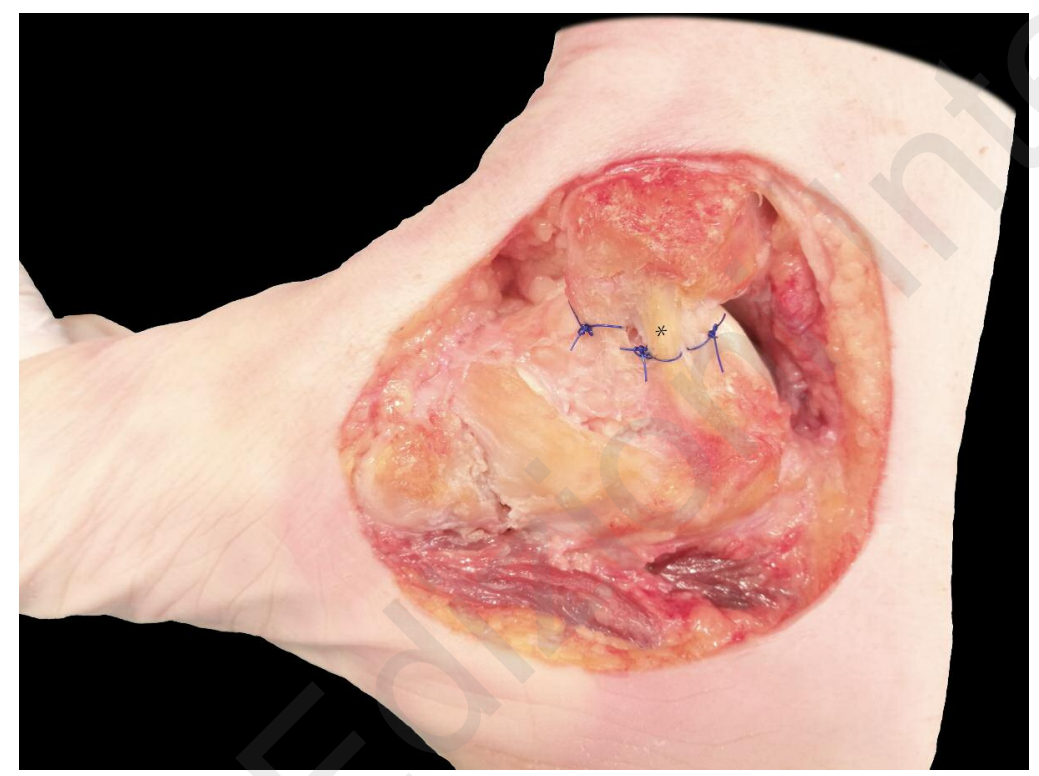

Figure 3. Dissection photographs showing the ligaments (tagged with suture) of the deltoid ligament deep layer. *The dITT ligament, a secondary bundle between the dATT ligament and dPTT ligament.

\section{Discussion}

Currently, there is common consensus in the literature that the $\mathrm{DL}$ is a ligamentous complex composed of different bands located in superficial and deep layers that attaches the medial malleolus to multiple bony areas of the medial ankle $4-6,10-15,21,24,28-34$. Conflicting evidence persists regarding the exact number and morphology of its bands $4,33,35$. Recently, a new interpretation of the medial collateral ligament complex was proposed by Cromeens ${ }^{24}$. Hence, the purpose of this cadaveric study was to evaluate the reproducibility of this new classification and investigate anatomical variations, to contribute in reaching an evidence-based consensus about the DL constituent bands. From a methodological point of view, in this study, as well as in several reports $21,25,26,35$, the SL was considered anatomically independent from the $\mathrm{DL}$ and not investigated. We believe that additional biomechanical and histological studies would be necessary to specifically investigate the connection between the SL and TCN ligament.

The complexity of the dissection of the DL and the difficulty of separating it from the joint capsule and tendon sheet of the posterior tibial and flexor digitorum longus muscles seems to explain in part the varied previous interpretations of its multiple ligamentous bands, identified during the years since early descriptions ${ }^{36,37}$. Sarrafian ${ }^{30}$ reported 13 different interpretations of the DL between 1822 and 1979, and up to 2016, at least 7 other interpretations have been published (Tab. II) 1,11-15,21,24,30,31. These include 8 different bands: the tibiocalcaneal ligament, the tibionavicular ligament, the anterior talotibial ligament, the 


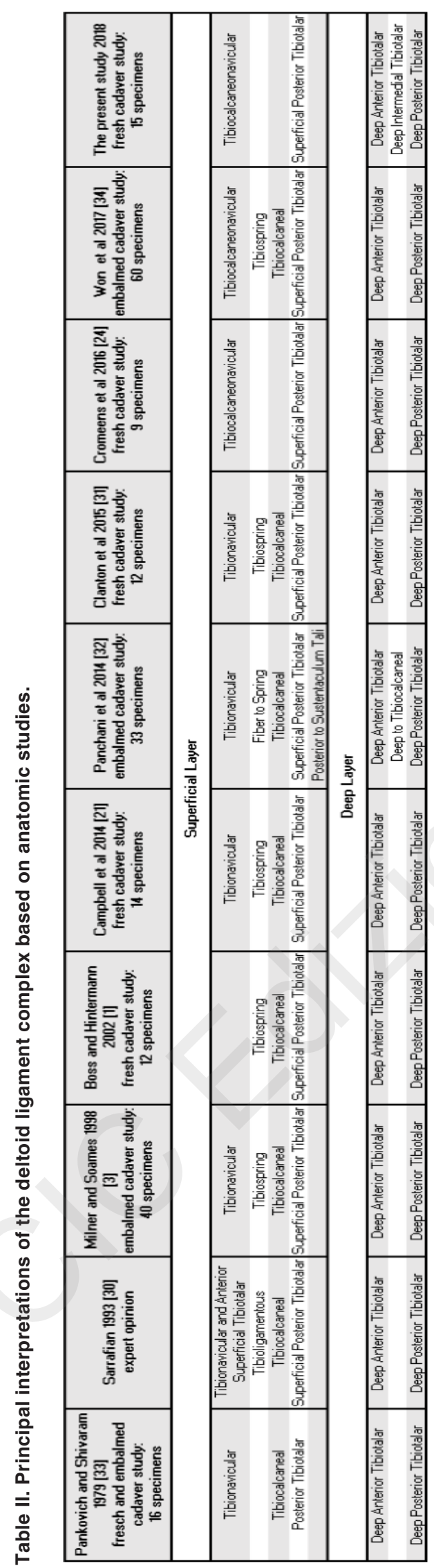

superficial posterior talotibial ligament, the deep posterior talotibial ligament, fibres to SL (plantar calcaneonavicular), a deep band to the tibiocalcaneal that attaches to the talus and a band posterior to the sustentaculum tali. In 1979, Pankovich and Shivaram ${ }^{33}$, after dissection of 16 cadaveric ankles, described 3 bands for the superficial layer (naviculotibial, calcaneotibial and superficial talotibial ligaments) and 2 for the deep layer (deep anterior talotibial and deep posterior talotibial ligaments). The Authors stated that the superficial layer attached to the navicular, the spring (plantar calcaneonavicular) ligament and the sustentaculum tali, while the fibres to the SL were not considered as a unique separate band ${ }^{32}$.

Currently, the most commonly accepted description of the DL is that proposed by Milner and Soames in $1998^{4}$, later almost entirely corroborated by Boss and Hintermann in $2002^{6}$. They distinguish six different components, named in accordance with their attachments. Four components belong to the superficial layer and two to the deep. They found that the tibiocalcaneal and tibiospring ligaments were the longest bands, and the tibiocalcaneal and posterior deep tibiotalar ligaments were the thickest. In 2003, Barnes described the appearance of the DL as sheet-like with a variable number of bands ${ }^{38}$. In our study, the TCN, the SPTT and the dPTT were consistently found, while the dATT ligament had a prevalence of $80 \%$. More recently, Yammine in his meta-analysis ${ }^{10}$ of cadaveric studies of DL anatomy demonstrated the prevalence of the different components proposed in the literature: the tibionavicular, the tibiospring and the tibiocalcaneal (bundles that we included in the TCN ligament) reached 90, 94 and $85 \%$, respectively; the sPTT ligament $80 \%$; while the dATT ligaments were the lowest with $63 \%$. In contrast, the dPTT ligament was always present (100\%).

Regarding the superficial layer, we considered the tibio navicular-spring-talar bundles as a unique anatomo-functional structure (the TCN ligament) including the traditional three ligaments: tibionavicular, tibiospring and tibiocalcaneal. We observed an insertional continuity of the components assuming a unique delta shaped fascial ligament with increasing thickness from the navicular tract to the spring-calcaneal tract. We considered further subdivisions of the TCN artificial and dependant on the dissection performed. TCN seems to play a major role in medial and rotatory stabilization of the ankle joint ${ }^{8,16}$ due to its bridging course to the talocrural, talocalcanel and talonavicolar joint and the longer momentum arm compared to the ligament of the deep layer ${ }^{34}$.

The tibionavicular part of the TCN ligament seems to restrain the talar head pronation as well as the talonavicular joint medial subluxation, playing an important role in preventing adult flatfoot deformity ${ }^{34}$. In agreement with several Authors $6,8,16,33,39$, in our study, the tibiocalcaneal component of TCN was found to be the thickest and strongest of all of the ligaments. To underline its functional importance, in the specimens where the tibiocalcaneal fibres were sec- 
tioned previously, Earll ${ }^{16}$ found a $26-43 \%$ decrease in tibiotalar contact areas and $20-30 \%$ increase in pressure peak. In contrast, when other bundles of DL were sectioned first, insignificant pressure changes were observed. In addition, Ziai et al. ${ }^{8}$ found in their biomechanical in vitro study that superficial layer lesion, in particular the tibiocalcaneal fibre lesion, was involved in rotatory instability, which increased when combined with lateral dual-ligament instability, while the deep layer played an insignificant role in rotatory instability after dissection.

The second component that we consistently found in the superficial layer was the SPTT ligament. It was the second longest ligament and the third largest, easily identified and widely described in the literature $4,6,8,24,40,41$ with various interpretations of its distal insertion. While some Authors 26,32 usually describe only its distal attachment to the medial talar tubercle, in our series, only 7 cases presented a single talus attachment, while the other 8 cases presented fibres crossing over the subtalar joint to the calcaneus. These findings are in agreement with those reported by Cromeens ${ }^{24}$, Boss ${ }^{6}$ and Milner ${ }^{4}$, who identified a calcaneal insertion of the SPTT ligament in the majority of their specimens $(6 / 9,9 / 9$ and $15 / 15$ respectively), suggesting that this ligament can play a secondary role on the stabilization of the subtalar joint. However, also the talar insertion was always found ${ }^{4,24}$ and described as the largest and widest distal insertion with respect to the calcaneal one. Hence, we believe that a multi-centre study with a larger number of specimens would be necessary to establish the proper nomenclature of this ligament in relation to its main insertion, naming it the superficial posterior tibio-talar or superficial posterior tibio-talo calcaneal ligament.

Cromeens ${ }^{24}$ proposed dividing the deep layer into two ligaments, the dATT and dPTT ligaments. In all our dissections, the dPTT ligament was easily identified in all specimens, while the dATT was not found in 3 specimens. A third ligament was isolated in three cases (20\% prevalence), the dITT ligament, always identified in association with a smaller dPTT ligament. This variant was previously reported by Sarrafian who considered the dITT ligament as a secondary bundle of the dPTT ligament ${ }^{8,16}$, while Panchani ${ }^{32} \mathrm{de}$ scribed a similar bundle that was named the deep to tibiocalcaneal ligament, with a prevalence in their specimens of $12 \%$. Boss described the dPTT ligament as the second strongest ligament after the tibicalcaneal bundle, while Woodburne ${ }^{42}$ and Panchani ${ }^{32}$ reported it as the thickest part of the medial ligament. In our series however, it was the third thickest after the TCN ligament with a thickness comparable to the SPTT ligament. In agreement with Cromeens, the dPTT ligament had the second largest proximal and distal insertion, suggesting that it remains the primary medial stabilizer of the talocrural joint. In our dissections, the dATT ligament was the smallest and thinnest ligament. As reported by Hyung-Jin Won ${ }^{34}$, it was located beneath the TCN and frequently blended with the ankle joint capsule, resulting difficult to isolate except by means of careful dissection under magnification.

Regarding gender, no statistically significant difference between males and females was found with respect to difference variables measured for each bundle (length, width and thickness), except for the length of the sPTT ligament $(p=0.0409)$. Considering the numerous variables analysed, this low statistical significance should be attributed to a random factor rather than to comparison factors. However, these results lead to the conclusion that the different study variables examined do not differ on average between the two sexes, demonstrating that the specimens of our samples are of the same population.

A major strength of this study is that, applying Cromeens's classification, it is possible to provide qualitative and quantitative observation of the morphology of the complex deltoid ligament. Further, our dissections were all performed with the supervision of an expert anatomist (R.M.) and all executed on fresh specimens, ensuring the most reliable measurements. However, our study has some limitations. First, 15 ankles specimens were included, all of Caucasian subjects, predominantly female and originally from the Iberian Peninsula, which are not fully representative of the variability of the entire population, particularly about measures such as length and thickness. Accordingly, our cohort was smaller than those used in the previous investigations by Milner and Soames ${ }^{4}$, whose study included 40 specimens, and Siegler ${ }^{41}$, whose study included 20 specimens; while it was bigger than those dissected by Campbell ${ }^{21}$ who recently reported the results of 14 specimens and the 9 considered by Cromeens ${ }^{24}$. Second, it was a purely descriptive anatomic study, without any testing of function. Multicentre studies would be necessary to increase the variability of the series, aiming to incorporate the anatomical study of the vascular supply and anatomy of the SL, an essential component of the medial ligament complex, which was not considered in this analysis.

In conclusion, throughout all of our meticulous dissections, Cromeens's attachment-based classification was proved to be simple, rational and reproducible for allowing a methodological description of the different bands of the DL. However, in our opinion, as well as in those of several Authors, the SL should be considered anatomically independent. By this recent classification, in the specimens dissected, it was possible to identify the following DL bands: the TCN, SPTT, dATT, dPTT and dITT ligaments. In particular, our findings showed that the dITT ligament must be considered as a secondary bundle of the dPTT ligament, which was not always identified in all specimens. Further, each DL component described in the current study has been so thoroughly detailed that the information presented can contribute, not only to reach an evidence-based consensus about its constituent bands, but also to develop proper anatomical repair and medial hindfoot ligament reconstruction proce- 
dures. Finally, further cadaveric and clinical studies are necessary to identify which are the most important ligamentous bands of the DL to repair or to reconstruct for ankle stability.

\section{Acknowledgements}

The Authors acknowledge Prof. M. Bolzan for his assistance with the statistical analysis.

\section{Conflict of interest statement}

The Authors declare that they have no conflict of interest related to the publication of this manuscript, and they have not received benefits or financial funds in support of this study.

\section{References}

1. Boss AP, Hintermann B. Anatomical study of the medial ankle ligament complex. Foot Ankle Int. 2002;23(6):547-553.

2. Kelikian AS, Sarrafian SK. Sarrafian's Anatomy of the Foot and Ankle: Descriptive, Topographic, Functional. Philadelphia, PA: Wolters Kluwer Health/Lippincott Williams \& Wilkins. 2011.

3. Milner CE, Soames RW. Anatomy of the collateral ligaments of the human ankle joint. Foot Ankle Int. 1998;19(11):757-760.

4. Milner CE, Soames RW. The medial collateral ligaments of the human ankle joint: anatomical variations. Foot Ankle Int. 1998;19(5):289-292.

5. Golanó P, Vega J, Pérez-Carro L, Götzens V. Ankle anatomy for the arthroscopist. Part II: Role of the ankle ligaments in soft tissue impingement. Foot Ankle Clin. 2006 Jun;11(2):275-296.

6. Boss AP, Hintermann B. Anatomical study of the medial ankle ligament complex. Foot Ankle Int. 2002 Jun;23(6):547-553.

7. Stufkens SA, van den Bekerom MP, Knupp M, Hintermann B, van $\mathrm{Dijk} C \mathrm{CN}$. The diagnosis and treatment of deltoid ligament lesions in supination-external rotation ankle fractures: a review. Strategies Trauma Limb Reconstr. 2012 Aug;7(2):73-85.

8. Ziai P, Benca E, Skrbensky GV, Wenzel F, Auffarth A, Krpo S, Windhager R, Buchhorn T. The role of the medial ligaments in lateral stabilization of the ankle joint: an in vitro study. Knee Surg Sports Traumatol Arthrosc. 2015 Jul;23(7):1900-1906.

9. Kopec TJ, Hibberd EE, Roos KG, Djoko A, Dompier TP, Kerr ZY. The Epidemiology of Deltoid Ligament Sprains in $25 \mathrm{Na}-$ tional Collegiate Athletic Association Sports, 2009-2010 Through 2014-2015 Academic Years. J Athl Train. 2017 Apr;52(4):350-359.

10. Yammine K. The Morphology and Prevalence of the Deltoid Complex Ligament of the Ankle. Foot Ankle Spec. 2017 Feb;10(1):55-62.

11. Savage-Elliott I, Murawski CD, Smyth NA, Golanó P, Kennedy JG. The deltoid ligament: an in-depth review of anatomy, function, and treatment strategies Knee Surg Sports Traumatol Arthrosc. 2013;21:1316-1327.

12. Mengiardi B, Pinto C, Zanetti M. Medial Collateral Ligament Complex of the Ankle: MR Imaging Anatomy and Findings in Medial Instability. Semin Musculoskelet Radiol. 2016 Feb;20(1):91-103.

13. Shiravi Z, Shadmehr A, Moghadam ST, Moghadam BA. Comparison of dynamic postural stability scores between athletes with and without chronic ankle instability during lateral jump landing. MLTJ. 2017 May 10;7(1):119-124.
14. Hintermann B, Knupp M, Pagenstert GI, Deltoid ligament injuries: diagnosis and management. Foot Ankle Clin. 2006 Sep;11(3):625-637.

15. Ferran NA, Oliva F, Maffulli N. Ankle instability. Sports Med Arthrosc. 2009 Jun;17(2):139-145.

16. Earll M, Wayne J, Brodrick C, Vokshoor A, Adelaar R. Contribution of the deltoid ligament to ankle joint contact characteristics: a cadaver study. Foot Ankle Int. 1996 Jun;17(6):317-324

17. Harper MC. An anatomic study of the short oblique fracture of the distal fibula and ankle stability. Foot Ankle. $1983 \mathrm{Jul}-$ Aug;4(1):23-29.

18. Waterman BR, Belmont PJ Jr, Cameron KL, Deberardino TM, Owens BD. Epidemiology of ankle sprain at the United States Military Academy. Am J Sports Med. 2010 Apr;38(4): 797-803.

19. Haddad SL, Dedhia S, Ren Y, Rotstein J, Zhang LQ. Deltoid ligament reconstruction: a novel technique with biomechanical analysis. Foot Ankle Int. $2010 \mathrm{Jul} ; 31(7): 639-651$.

20. Vega J, Allmendinger J, Malagelada F, Guelfi M, Dalmau-Pastor M. Combined arthroscopic all-inside repair of lateral and medial ankle ligaments is an effective treatment for rotational ankle instability. Knee Surg Sports Traumatol Arthrosc. 2017 Oct 5. doi: 10.1007/s00167-017-4736-y.

21. Campbell KJ, Michalski MP, Wilson KJ, Goldsmith MT, Wijdicks CA, LaPrade RF, Clanton TO, The ligament anatomy of the deltoid complex of the ankle: a qualitative and quantitative anatomical study.J Bone Joint Surg Am. 2014 Apr 16;96(8):e62.

22. Wenny R, Duscher D, Meytap E, Weninger P, Hirtler L. Dimensions and attachments of the ankle ligaments: evaluation for ligament reconstruction. Anat Sci Int. 2015 Jun;90(3):161171.

23. Terminologia anatomica: international anatomical terminology, Federative committee on anatomical terminology (1998) Stuttgart. New York: Thieme. 1998.

24. Cromeens BP, Kirchhoff CA, Patterson RM, Motley T, Stewart D, Fisher C, Reeves RE. An attachment-based desription of the medial collateral and spring ligament complexes. Foot Ankle Int. 2015 Jun;36(6):710-721.

25. Alireza Mousavian, MD, Jakrapong Orapin, MD, Apisan Chinanuvathana, MD, Lew C. Schon, Anatomic Spring Ligament and Posterior Tibial Tendon Reconstruction: New Concept of Double Bundle PTT and a Novel Technique for Spring Ligament. Arch Bone Jt Surg. 2017 May;5(3):201-205.

26. Mengiardi B, Pinto C, Zanetti M.Spring Ligament Complex and Posterior Tibial Tendon: MR Anatomy and Findings in Acquired Adult Flatfoot Deformity. Semin Musculoskelet Radiol. 2016 Feb;20(1):104-115.

27. Padulo J, Oliva F, Frizziero A, Maffulli N. Muscles, Ligaments and Tendons Journal - Basic principles and recommendations in clinical and field science research: 2016 update. MLTJ. 2016;6(1):1-5.

28. Agnihotri AK, Purwar B, Googoolye K, Agnihotri S, Jeebun N Estimation of stature by foot length. J Forensic Leg Med. 2007 Jul;14(5):279-283.

29. Standring S. Gray's Anatomy: The Anatomical Basis of Clinical Practice. 40th ed. Oxford, UK: Churchill Livingstone. 2008.

30. Sarrafian SK, ed. Anatomy of the Foot and Ankle. Descriptive, Topographic, Functional. 2nd ed. Philadelphia, PA: JB Lippincott. 1993:159-217.

31. Clanton TO, Williams BT, James EW, Campbell KJ, Rasmussen MT, Haytmanek CT, Wijdicks CA, LaPrade RF. Radiographic Identification of the Deltoid Ligament Complex of the Medial Ankle. Am J Sports Med. 2015 Nov;43(11):27532762.

32. Panchani PN, Chappell TM, Moore GD, Tubbs RS, Shoja MM, Loukas M, Kozlowski PB, Khan KH, DiLandro AC, D'A toni AV. Anatomic study of the deltoid ligament of the ankle. Foot Ankle Int. 2014;35(9):916-921. 
33. Pankovich AM, Shivaram MS. Anatomical basis of variability in injuries of the medial malleolus and the deltoid ligament. II. Clinical studies. Acta Orthop Scand. 1979;50(2):225-236.

34. Won HJ, Koh IJ, Won HS. Morphological variations of the deltoid ligament of the medial ankle. Clin Anat. 2016 Nov; 29(8):1059-1065.

35. Golano P, Vega J, de Leeuw PA, et al. Anatomy of the ankle ligaments: a pictorial essay. Knee Surg Sports Traumatol Arthros. 2010;18(5):557-569.

36. Toldt C. Anatomischer Atlas für Studierende und Ärzte unter mitwirkung von Alois Dalla Rosa. 11th ed. Berlin: Urban \& Schwarzenberg. German. 1921

37. Poirier P, Charpy A. Traité d'Anatomie Humaine. Paris,
France: Masson. 1899.

38. Barnes DJ. Anatomy of Lower Extremity. Marietta, OH: CBLS. 2003.

39. Siegler S, Block J and Schneck CD: The mechanical characteristics of the collateral ligaments of the human anklejoint. Foot Ankle. 1988 Apr;8(5):234-242.

40. Hintermann B, Golanò P. The Anatomy and Function of the Deltoid Ligament. Tecniques in Foot \& Ankle Surgery. 2014 Jun;13(2):67-72.

41. Williams PL, Bannister LH, Berry MM, et al. Gray's Anatomy, 38th Ed. London, Churchill Livingstone. 1995:712-722.

42. Woodburne RT, Burkel WE. Essentials of human anatomy. 8th Ed. New York: Oxford University Press. 1988:642-643. 